\title{
natureINSIGH
}

\section{NEURODEGENERATIVE DISEASES}

10 November 2016 / Vol 539 / Issue No 7628

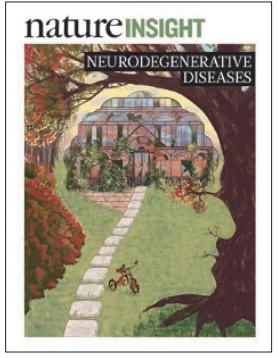

Cover illustration Nik Spencer

Editor, Nature

Philip Campbell

Publishing

Richard Hughes

Insights Editor

Ursula Weiss

Production Editor

Elizabeth Batty

Art Editor

Nik Spencer

Sponsorship

Janice Stevenson

Samantha Meyer

Production

lan Pope

Marketing

Nicole Jackson

Editorial Assistant

Giacomo Russo

The Campus

4 Crinan Street

London N1 9XW, UK

Tel: +44 (0) 2078334000

e: nature@nature.com
L

The prevalence of neurodegenerative disorders is increasing, owing - in part - to extensions in

lifespan. Currently, there is no cure for any of these diseases, although not for lack of trying. The hard work and dedication that goes into unravelling mechanisms of disease is discernible from this collection of reviews. Each summarises our knowledge, highlights exciting advances and provides ample inspiration for future research.

The signs of the passage of time are clearly visible in the brain. Tony Wyss-Coray synthesizes current knowledge on brain ageing and neurodegeneration and explores the prospect of stalling, or even resetting, the clock.

Growing evidence suggests that genetic, cellular and circuit dysregulation results from, and can lead to, cellular and cognitive hallmarks of Alzheimer's disease. Li-Huei Tsai, Rebecca Canter and Jay Penney argue for a multipronged approach to the treatment of this common form of dementia.

Paul Taylor, Robert Brown and Don Cleveland discuss emerging themes and mechanisms that underlie amyotrophic lateral sclerosis (also known as Lou Gehrig's disease or motor neuron disease), a progressive degeneration of motor neurons in the brain and spinal cord.

Parkinson's disease is characterized by the progressive death of dopamine neurons. Asa Abeliovich and Aaron Gitler propose that the accumulation of cellular damage eventually overwhelms the protein-disposal mechanisms of these neurons.

John Collinge considers the wider relevance of mammalian prions for neurodegenerative diseases. And Roland Riek and David Eisenberg provide a structural perspective on neurodegeneration through the properties of protein aggregates, the hallmarks of various neurodegenerative disorders. They explore the self-replication, cell-to-cell transmission and toxicity of these amyloids.

We hope that this collection will not only stimulate further research on neurodegenerative diseases but also direct more funding towards this area - as a greater understanding will reveal new opportunities for therapeutic intervention.

Nature is pleased to acknowledge the financial support of Eli Lilly and Company in producing this Insight. As always, Nature carries sole responsibility for all editorial content.

\section{CONTENTS}

\section{REVIEWS}

180 Ageing, neurodegeneration and brain rejuvenation

Tony Wyss-Coray

187 The road to restoring neural circuits for the treatment of Alzheimer's disease

Rebecca G. Canter, Jay Penney \& Li-Huei Tsai

197 Decoding ALS: from genes to mechanism

J. Paul Taylor, Robert H. Brown Jr \& Don W. Cleveland

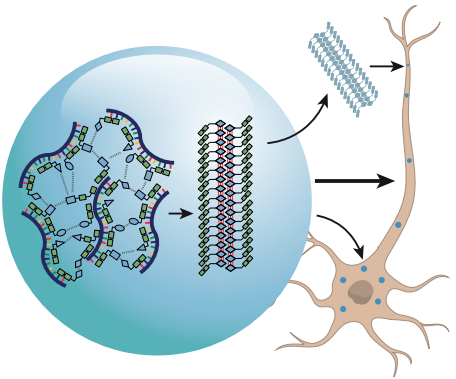

207 Defects in trafficking bridge Parkinson's disease pathology and genetics

Asa Abeliovich \& Aaron D. Gitler

217 Mammalian prions and their wider relevance in neurodegenerative diseases John Collinge

227 The activities of amyloids from a structural perspective Roland Riek \& David S. Eisenberg

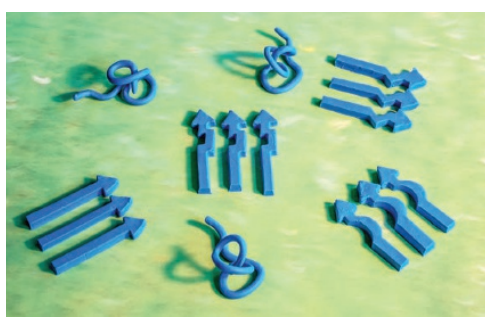

